\title{
Analysis of particulate emissions from tropical biomass burning using a global aerosol model and long-term surface observations
}

\section{Carly L. Reddington et al.}

Correspondence to: C. L. Reddington (c.1.s.reddington@leeds.ac.uk)

The copyright of individual parts of the supplement might differ from the CC-BY 3.0 licence. 


\section{S1. Description of the k-Köhler water uptake scheme}

In Sect. 4.1.3., we test the sensitivity of the simulated aerosol optical depth (AOD) to the calculation of water uptake by using the $\kappa-$ Köhler water uptake scheme, based upon the Köhler equation with a single parameter, $\kappa$, defining the water uptake for different chemical species (Petters and Kreidenweis, 2007). The species-dependent hygroscopiticy parameter, $\kappa$, is defined through its effect upon the water activity of the solution as follows:

$$
\frac{1}{a_{w}}=1+\kappa \frac{V_{s}}{V_{w}}
$$

Where $V_{s}$ is the volume of the dry aerosol and $V_{w}$ is the volume of water. Using Köhler theory and the above equation the relationship between the relative humidity and the growth of the aerosol can be defined as follows (see Petters and Kreidenweis, 2007, for derivation):

$$
S(D)=\frac{D^{3}-D_{d}^{3}}{D^{3}-D_{d}^{3}(1-\kappa)} \exp \left(\frac{4 \sigma_{s / a} M_{w}}{R T \rho_{w} D}\right),
$$

where $S$ is the saturation ratio, $D_{d}$ is the dry diameter, $D$ is the wet diameter, $\kappa$ is the hygroscopic parameter specific to the solute, $\sigma_{s / a}$ is the surface tension of the droplet, $R$ is the universal gas constant, $T$ is the temperature and $M_{w}$ and $\rho_{w}$ are the molecular mass and density of water, respectively. In the model this equation is solved iteratively by incrementing $D$ until the saturation ratio is equal to the ambient relative humidity. The growth factor and volume of water can be determined from this and used to calculate the refractive index of the wetted aerosol.

\section{S2. Evaluation of simulated mass extinction efficiency}

Reid and Hobbs (1998) report values of mass absorption efficiency (MAE) for smouldering $\left(0.7 \pm 0.1 \mathrm{~m}^{2} \mathrm{~g}^{-1}\right)$ and flaming $\left(1.0 \pm 0.2 \mathrm{~m}^{2} \mathrm{~g}^{-1}\right)$ forest fires in Brazil, sampled between $13^{\text {th }}$ August and $25^{\text {th }}$ September 1995. To evaluate the simulated mass extinction efficiency (MEE) against observations, we calculated values of MEE from the observed MAE and single scattering albedo (SSA) from Reid and Hobbs (1998), assuming: MAE = MEE * (1-SSA). For smouldering forest fires we obtained an "observed" MEE (550 nm) of $4.4 \mathrm{~m}^{2} \mathrm{~g}^{-1}$ (range: 3.3 to $5.7 \mathrm{~m}^{2} \mathrm{~g}^{-1}$, calculated from the quoted standard errors). To compare to the observed value, we calculated MEEs at 550 $\mathrm{nm}$ for each simulation (with fire emissions), in grid cells that cover the locations where smoke from the forest fires were sampled (in the vicinity of Porto Velho, Rondônia and Marabá, Pará), and calculated an average for August over the period 2003-2011. 
The average simulated MEE values of 5.2-5.4 $\mathrm{m}^{2} \mathrm{~g}^{-1}$ (using the ZSR water uptake scheme to calculate aerosol hygroscopic growth) and 3.5-3.6 $\mathrm{m}^{2} \mathrm{~g}^{-1}$ (using the $\kappa$-Köhler water uptake scheme) span the observed value and are within the uncertainty range of the observations. The range in the simulated values (e.g. $5.18-5.35 \mathrm{~m}^{2} \mathrm{~g}^{-1}$ ) demonstrates the relatively limited sensitivity of the MEE to the fire emission dataset (average values are within 5\%) compared to the sensitivity to the calculation of aerosol hygroscopic growth (with average values differing by a factor of 1.5). The comparison between simulated and observed MEEs supports the conclusion in the main text (Sect. 4.1.3) that the ZSR and $\kappa$-Köhler AOD are likely to represent high and low water uptake cases, respectively.

We also compare the GLOMAP simulated global mean values for aerosol burden, AOD, and MEE against those of other global aerosol models (see Table S2). In general we find that the GLOMAP global mean aerosol burdens and AOD $(550 \mathrm{~nm})$ are consistent with values from AEROCOM (Kinne et al., 2006) and Heald et al. (2014) for $\mathrm{SO}_{4}, \mathrm{BC}$ and sea salt. For the POM and mineral dust components, both the burden and AOD are underestimated by GLOMAP relative to the other models. There could be several reasons for this underestimation (including different anthropogenic emissions and/or aerosol removal schemes in the models), but one factor that may partly explain the higher burden and AOD values for POM from the GEOS-Chem model relative to GLOMAP is the higher assumed POM:OC ratio of 2 (Heald et al., 2014), compared to 1.4 assumed in GLOMAP. The GLOMAP simulated global mean MEEs for all components are within the large range in values reported by AEROCOM (Kinne et al., 2006; Mhyre et al., 2013) and Heald et al. (2014). The MEEs for POM, $\mathrm{SO}_{4}$ and $\mathrm{BC}$ calculated using the ZSR water uptake scheme are generally at the upper end of the AEROCOM values (particularly for BC), and those calculated using the $\kappa$-Köhler water uptake scheme are towards the lower end. 
Table S1. Summary of the AERONET and particulate matter (PM) measurement stations used to evaluate the model. The geographical positions of the stations are listed as: latitude, longitude, elevation above sea level. The Principal Investigator(s) (PI) responsible for each dataset are shown in the final column. Ascension Island is listed with the African sites because it samples outflow of biomass burning aerosol from the African continent (Swap et al., 1996). When comparing the model and observations, we restrict the time period to between January 2003 and December 2011 (according to the availability of biomass burning emissions data).

\begin{tabular}{|c|c|c|c|c|c|}
\hline Station & Country & $\begin{array}{l}\text { Observation } \\
\text { period }\end{array}$ & $\begin{array}{l}\text { Geographical } \\
\text { position }\end{array}$ & $\begin{array}{l}\text { Location } \\
\text { classification }\end{array}$ & PI \\
\hline \multicolumn{6}{|l|}{ South America } \\
\hline $\begin{array}{l}\text { Porto Velho } \\
\text { (PM2.5) }\end{array}$ & $\begin{array}{l}\text { Rondonia, } \\
\text { Brazil }\end{array}$ & $\begin{array}{l}25 / 09 / 09- \\
04 / 10 / 12\end{array}$ & $\begin{array}{l}8.687^{\circ} \mathrm{S}, \\
63.867^{\circ} \mathrm{W}, \\
94.0 \mathrm{~m} \\
\end{array}$ & $\begin{array}{l}\text { Heavily impacted } \\
\text { by biomass } \\
\text { burning }\end{array}$ & Paulo Artaxo \\
\hline $\begin{array}{l}\text { Alta Floresta } \\
\text { (PM } 2.5)\end{array}$ & $\begin{array}{l}\text { Mato Grosso, } \\
\text { Brazil }\end{array}$ & $\begin{array}{l}24 / 08 / 92- \\
06 / 03 / 05\end{array}$ & $\begin{array}{l}9.871^{\circ} \mathrm{S}, \\
56.104^{\circ} \mathrm{W} \\
277.0 \mathrm{~m}\end{array}$ & $\begin{array}{l}\text { Rural, city } \\
\text { outskirts, heavily } \\
\text { impacted by } \\
\text { biomass burning }\end{array}$ & Paulo Artaxo \\
\hline $\begin{array}{l}\text { Manaus, TT34 } \\
\text { tower } \\
\text { (PM2.5) }\end{array}$ & $\begin{array}{l}\text { INPA Cuieiras } \\
\text { forest reserve, } \\
\text { Amazonas, } \\
\text { Brazil }\end{array}$ & $\begin{array}{l}10 / 02 / 08- \\
25 / 10 / 11\end{array}$ & $\begin{array}{l}2.594^{\circ} \mathrm{S} \\
60.209^{\circ} \mathrm{W} \\
110.0 \mathrm{~m}\end{array}$ & Preserved forest & Paulo Artaxo \\
\hline $\begin{array}{l}\text { Santarem, K67 } \\
\text { tower } \\
\left(\mathbf{P M}_{2.5}\right)\end{array}$ & $\begin{array}{l}\text { Tapajos } \\
\text { National } \\
\text { Forest, Para, } \\
\text { Brazil }\end{array}$ & $\begin{array}{l}04 / 02 / 00- \\
01 / 04 / 07\end{array}$ & $\begin{array}{l}2.850^{\circ} \mathrm{S} \\
54.867^{\circ} \mathrm{W} \\
70.0 \mathrm{~m}\end{array}$ & Preserved Forest & Paulo Artaxo \\
\hline $\begin{array}{l}\text { Alta Floresta } \\
\text { (AERONET) }\end{array}$ & $\begin{array}{l}\text { Mato Grosso, } \\
\text { Brazil }\end{array}$ & $\begin{array}{l}21 / 06 / 93- \\
15 / 01 / 14\end{array}$ & $\begin{array}{l}9.871^{\circ} \mathrm{S}, \\
56.104^{\circ} \mathrm{W}, \\
277.0 \mathrm{~m}\end{array}$ & $\begin{array}{l}\text { Rural, town } \\
\text { outskirts }\end{array}$ & $\begin{array}{l}\text { Brent Holben, } \\
\text { Paulo Artaxo }\end{array}$ \\
\hline $\begin{array}{l}\text { Cuiaba Miranda } \\
\text { (AERONET) }\end{array}$ & $\begin{array}{l}\text { Mato Grosso, } \\
\text { Brazil }\end{array}$ & $\begin{array}{l}23 / 03 / 01 \\
07 / 10 / 13\end{array}$ & $\begin{array}{l}15.730^{\circ} \mathrm{S}, \\
56.021^{\circ} \mathrm{W}, \\
210.0 \mathrm{~m}\end{array}$ & $\begin{array}{l}\text { Rural, city } \\
\text { outskirts }\end{array}$ & $\begin{array}{l}\text { P. Artaxo, J. de } \\
\text { Souza Nogueira, E. } \\
\text { Ojeda de Almeida } \\
\text { Filho, A. Jorge }\end{array}$ \\
\hline $\begin{array}{l}\text { Rio Branco } \\
\text { (AERONET) }\end{array}$ & Acre, Brazil & $\begin{array}{l}02 / 07 / 00- \\
10 / 11 / 12\end{array}$ & $\begin{array}{l}9.957^{\circ} \mathrm{S}, \\
67.869^{\circ} \mathrm{W}, \\
212.0 \mathrm{~m} \\
\end{array}$ & $\begin{array}{l}\text { Urban, within city } \\
\text { limits }\end{array}$ & $\begin{array}{l}\text { Brent Holben, } \\
\text { Paulo Artaxo }\end{array}$ \\
\hline $\begin{array}{l}\text { Ji Parana SE } \\
\text { (AERONET) }\end{array}$ & $\begin{array}{l}\text { Rondonia, } \\
\text { Brazil }\end{array}$ & $\begin{array}{l}19 / 01 / 06- \\
09 / 06 / 13\end{array}$ & $\begin{array}{l}10.934^{\circ} \mathrm{S} \\
61.852^{\circ} \mathrm{W} \\
218.0 \mathrm{~m}\end{array}$ & Rural & Paulo Artaxo \\
\hline $\begin{array}{l}\text { Abracos Hill } \\
\text { (AERONET) }\end{array}$ & $\begin{array}{l}\text { Rondonia, } \\
\text { Brazil }\end{array}$ & $\begin{array}{l}22 / 01 / 99- \\
09 / 10 / 05\end{array}$ & $\begin{array}{l}10.760^{\circ} \mathrm{S} \\
62.358^{\circ} \mathrm{W} \\
200.0 \mathrm{~m}\end{array}$ & Rural & $\begin{array}{l}\text { Brent Holben, } \\
\text { Paulo Artaxo }\end{array}$ \\
\hline $\begin{array}{l}\text { Belterra } \\
\text { (AERONET) }\end{array}$ & Para, Brazil & $\begin{array}{l}21 / 09 / 99- \\
24 / 04 / 05\end{array}$ & $\begin{array}{l}2.648^{\circ} \mathrm{S}, \\
54.952^{\circ} \mathrm{W}, \\
70.0 \mathrm{~m}\end{array}$ & Rural & $\begin{array}{l}\text { B. Holben, P. } \\
\text { Artaxo }\end{array}$ \\
\hline $\begin{array}{l}\text { Santa Cruz } \\
\text { (AERONET) }\end{array}$ & Bolivia & $\begin{array}{l}20 / 01 / 99- \\
26 / 11 / 13\end{array}$ & $\begin{array}{l}17.802^{\circ} \mathrm{S}, \\
63.178^{\circ} \mathrm{W} \\
442.0 \mathrm{~m}\end{array}$ & Urban, city centre & B. Holben \\
\hline $\begin{array}{l}\text { Santa Cruz } \\
\text { UTESPA } \\
\text { (AERONET) }\end{array}$ & Bolivia & $\begin{array}{l}18 / 09 / 06- \\
04 / 11 / 08\end{array}$ & $\begin{array}{l}17.767^{\circ} \mathrm{S} \\
63.201^{\circ} \mathrm{W} \\
432.0 \mathrm{~m}\end{array}$ & $\begin{array}{l}\text { Urban, within city } \\
\text { limits }\end{array}$ & B. Holben \\
\hline \multicolumn{6}{|c|}{ Equatorial Asia \& Philippines } \\
\hline $\begin{array}{l}\text { Singapore } \\
\text { (AERONET) }\end{array}$ & Singapore & $\begin{array}{l}14 / 11 / 06- \\
19 / 10 / 12\end{array}$ & $\begin{array}{l}103.780^{\circ} \mathrm{E}, \\
1.298^{\circ} \mathrm{N}, \\
30.0 \mathrm{~m}\end{array}$ & Urban, city centre & $\begin{array}{l}\text { S.-C. Liew, S. V. } \\
\text { Salinas Cortijo }\end{array}$ \\
\hline
\end{tabular}




\begin{tabular}{|c|c|c|c|c|c|}
\hline $\begin{array}{l}\text { Bandung } \\
\text { (AERONET) }\end{array}$ & $\begin{array}{l}\text { Java, } \\
\text { Indonesia }\end{array}$ & $\begin{array}{l}13 / 05 / 09- \\
28 / 09 / 11\end{array}$ & $\begin{array}{l}107.61^{\circ} \mathrm{E} \\
6.888^{\circ} \mathrm{N} \\
826.0 \mathrm{~m}\end{array}$ & Urban, city centre & $\begin{array}{l}\text { P. Lestari, B. } \\
\text { Holben }\end{array}$ \\
\hline $\begin{array}{l}\text { Manila } \\
\text { Observatory } \\
\text { (AERONET) }\end{array}$ & $\begin{array}{l}\text { Quezon City, } \\
\text { Philippines }\end{array}$ & $\begin{array}{l}21 / 01 / 09- \\
30 / 12 / 11\end{array}$ & $\begin{array}{l}121.08^{\circ} \mathrm{E} \\
14.635^{\circ} \mathrm{N} \\
63.0 \mathrm{~m}\end{array}$ & $\begin{array}{l}\text { Urban, within city } \\
\text { limits }\end{array}$ & $\begin{array}{l}\text { N. Lagrosas, B. } \\
\text { Holben }\end{array}$ \\
\hline $\begin{array}{l}\text { ND Marbel Univ. } \\
\text { (AERONET) }\end{array}$ & $\begin{array}{l}\text { Koronadal, } \\
\text { Philippines }\end{array}$ & $\begin{array}{l}17 / 12 / 09- \\
19 / 01 / 12\end{array}$ & $\begin{array}{l}124.843^{\circ} \mathrm{E}, \\
6.496^{\circ} \mathrm{N} \\
70.0 \mathrm{~m} \\
\end{array}$ & Urban, city centre & S. Dorado \\
\hline \multicolumn{6}{|l|}{ Indochina } \\
\hline $\begin{array}{l}\text { Songkhla Met. } \\
\text { Station } \\
\text { (AERONET) }\end{array}$ & Thailand & $\begin{array}{l}11 / 01 / 07- \\
13 / 12 / 11\end{array}$ & $\begin{array}{l}100.61^{\circ} \mathrm{E}, \\
7.184^{\circ} \mathrm{N} \\
15.0 \mathrm{~m}\end{array}$ & Urban, city centre & S. Janjai \\
\hline $\begin{array}{l}\text { Chulalongkorn } \\
\text { (AERONET) }\end{array}$ & Thailand & $\begin{array}{l}19 / 02 / 03- \\
25 / 09 / 04\end{array}$ & $\begin{array}{l}100.53^{\circ} \mathrm{E} \\
13.736^{\circ} \mathrm{N} \\
115.0 \mathrm{~m}\end{array}$ & Urban, city centre & B. Holben \\
\hline $\begin{array}{l}\text { Ubon } \\
\text { Ratchathani } \\
\text { (AERONET) } \\
\end{array}$ & Thailand & $\begin{array}{l}09 / 10 / 09- \\
07 / 11 / 12\end{array}$ & $\begin{array}{l}104.87^{\circ} \mathrm{E}, \\
15.246^{\circ} \mathrm{N}, \\
120.0 \mathrm{~m} \\
\end{array}$ & $\begin{array}{l}\text { Urban, within city } \\
\text { limits }\end{array}$ & S. Janjai \\
\hline $\begin{array}{l}\text { Silpakorn } \\
\text { University } \\
\text { (AERONET) }\end{array}$ & Thailand & $\begin{array}{l}15 / 08 / 06- \\
11 / 12 / 11\end{array}$ & $\begin{array}{l}100.04^{\circ} \mathrm{E}, \\
13.819^{\circ} \mathrm{N}, \\
72.0 \mathrm{~m}\end{array}$ & $\begin{array}{l}\text { Urban, city } \\
\text { outskirts }\end{array}$ & S. Janjai \\
\hline $\begin{array}{l}\text { Chiang Mai Met. } \\
\text { Station } \\
\text { (AERONET) }\end{array}$ & Thailand & $\begin{array}{l}\text { 17/09/06 - } \\
28 / 07 / 11\end{array}$ & $\begin{array}{l}98.973^{\circ} \mathrm{E}, \\
18.771^{\circ} \mathrm{N}, \\
312.0 \mathrm{~m} \\
\end{array}$ & $\begin{array}{l}\text { Urban, city } \\
\text { outskirts }\end{array}$ & S. Janjai \\
\hline $\begin{array}{l}\text { Phimai } \\
\text { (AERONET) }\end{array}$ & Thailand & $\begin{array}{l}18 / 02 / 03- \\
10 / 04 / 08\end{array}$ & $\begin{array}{l}102.56^{\circ} \mathrm{E}, \\
15.182^{\circ} \mathrm{N}, \\
220.0 \mathrm{~m} \\
\end{array}$ & Rural & B. Holben \\
\hline $\begin{array}{l}\text { Mukdahan } \\
\text { (AERONET) }\end{array}$ & Thailand & $\begin{array}{l}07 / 11 / 03- \\
30 / 05 / 10\end{array}$ & $\begin{array}{l}104.68^{\circ} \mathrm{E}, \\
16.607^{\circ} \mathrm{N}, \\
166.0 \mathrm{~m} \\
\end{array}$ & Rural & Brent Holben \\
\hline $\begin{array}{l}\text { Bac Giang } \\
(\text { AERONET) }\end{array}$ & Vietnam & $\begin{array}{l}03 / 03 / 03- \\
26 / 12 / 09\end{array}$ & $\begin{array}{l}106.23^{\circ} \mathrm{E}, \\
21.291^{\circ} \mathrm{N}, \\
15.0 \mathrm{~m}\end{array}$ & $\begin{array}{l}\text { Rural, city } \\
\text { outskirts }\end{array}$ & N. Xuan Anh \\
\hline $\begin{array}{l}\text { Bac Lieu } \\
\text { (AERONET) }\end{array}$ & Vietnam & $\begin{array}{l}10 / 03 / 03- \\
25 / 04 / 11\end{array}$ & $\begin{array}{l}105.73^{\circ} \mathrm{E} \\
9.280^{\circ} \mathrm{N} \\
10.0 \mathrm{~m} \\
\end{array}$ & $\begin{array}{l}\text { Rural, city } \\
\text { outskirts }\end{array}$ & N. Xuan Anh \\
\hline \multicolumn{6}{|l|}{ Africa } \\
\hline $\begin{array}{l}\text { Ilorin } \\
\text { (AERONET) }\end{array}$ & Ilorin, Nigeria & $\begin{array}{l}25 / 04 / 98- \\
13 / 09 / 14\end{array}$ & $\begin{array}{l}8.320^{\circ} \mathrm{N} \\
4.340^{\circ} \mathrm{E}, \\
350.0 \mathrm{~m}\end{array}$ & $\begin{array}{l}\text { Rural, heavily } \\
\text { impacted by dust } \\
\text { emissions }\end{array}$ & R. T. Pinker \\
\hline $\begin{array}{l}\text { ICIPE-Mbita } \\
\text { (AERONET) }\end{array}$ & Mbita, Kenya & $\begin{array}{l}20 / 03 / 06- \\
10 / 04 / 14\end{array}$ & $\begin{array}{l}0.417^{\circ} \mathrm{S}, \\
34.200^{\circ} \mathrm{E}, \\
1125.0 \mathrm{~m} \\
\end{array}$ & $\begin{array}{l}\text { Sub-urban/rural, } \\
\text { coastal Lake } \\
\text { Victoria }\end{array}$ & B. Holben \\
\hline $\begin{array}{l}\text { Mongu } \\
\text { (AERONET) }\end{array}$ & $\begin{array}{l}\text { Mongu, } \\
\text { Zambia }\end{array}$ & $\begin{array}{l}27 / 06 / 95- \\
15 / 01 / 10\end{array}$ & $\begin{array}{l}15.254^{\circ} \mathrm{S} \\
23.151^{\circ} \mathrm{E} \\
1107.0 \mathrm{~m}\end{array}$ & $\begin{array}{l}\text { Urban, within city } \\
\text { limits }\end{array}$ & B. Holben \\
\hline $\begin{array}{l}\text { Skuzkuza } \\
\text { (AERONET) }\end{array}$ & $\begin{array}{l}\text { Skukuza, } \\
\text { South Africa }\end{array}$ & $\begin{array}{l}19 / 07 / 98- \\
03 / 08 / 11\end{array}$ & $\begin{array}{l}24.992^{\circ} \mathrm{S}, \\
31.588^{\circ} \mathrm{E}, \\
150.0 \mathrm{~m}\end{array}$ & Rural & $\begin{array}{l}\text { B. Holben, S. } \\
\text { Piketh }\end{array}$ \\
\hline $\begin{array}{l}\text { Wits University } \\
\text { (AERONET) }\end{array}$ & $\begin{array}{l}\text { Johannesburg, } \\
\text { South Africa }\end{array}$ & $\begin{array}{l}09 / 05 / 02- \\
14 / 11 / 11\end{array}$ & $\begin{array}{l}26.192^{\circ} \mathrm{S} \\
28.029^{\circ} \mathrm{E} \\
1775.0 \mathrm{~m}\end{array}$ & Urban, city centre & S. Piketh \\
\hline $\begin{array}{l}\text { Ascension Island } \\
\text { (AERONET) }\end{array}$ & $\begin{array}{l}\text { Ascension } \\
\text { Island }\end{array}$ & $\begin{array}{l}20 / 11 / 98- \\
31 / 12 / 13\end{array}$ & $\begin{array}{l}7.976^{\circ} \mathrm{S}, \\
14.414^{\circ} \mathrm{W}, \\
30.0 \mathrm{~m} \\
\end{array}$ & $\begin{array}{l}\text { Island/coastal, } \\
\text { Atlantic Ocean }\end{array}$ & B. Holben \\
\hline
\end{tabular}


Table S2. Simulated global annual mean (volume-weighted) aerosol budget, aerosol optical depth (AOD) at $550 \mathrm{~nm}$ and mass extinction efficiency (MEE) for a 2010 GLOMAP simulation with GFED3 fire emissions. GLOMAP simulated AOD and MEE values are shown for two different methods of calculating the aerosol hygroscopic growth: the ZSR and $\kappa$-Köhler water uptake schemes (described in the text).

\begin{tabular}{|c|c|c|c|}
\hline & Burden / Tg & $\mathrm{AOD}, 550 \mathrm{~nm}$ & $\mathrm{MEE} / \mathrm{m}^{2} \mathrm{~g}^{-1}$ \\
\hline & & ZSR $\quad \kappa-$ Köhler & ZSR $\quad \kappa-$ Köhler \\
\hline Sulphate & 2.02 & 0.0186 & 10.1 \\
\hline & {$\left[1.27^{\mathrm{a}}, 1.99^{\mathrm{b}}\right]$} & {$\left[0.0154^{\mathrm{a}}, 0.034^{\mathrm{b}}\right]$} & {$\left[6.3^{\mathrm{a}}, 8.5^{\mathrm{b}}\right],(12.7 \pm 8.6)^{\mathrm{c}}$} \\
\hline $\mathrm{BC}$ & 0.11 & $\begin{array}{ll}0.0023 & 0.0017\end{array}$ & 14.1 \\
\hline & {$\left[0.10^{\mathrm{a}}, 0.20^{\mathrm{b}}\right]$} & {$\left[0.0012^{\mathrm{a}}, 0.004^{\mathrm{b}}\right]$} & {$\left[5.9^{\mathrm{a}}, 8.9^{\mathrm{b}}\right],(10.5 \pm 3.9)^{\mathrm{c}}$} \\
\hline POM & 0.99 & 0.0132 & 8.8 \\
\hline & {$\left[2.01^{\mathrm{a}}, 1.68^{\mathrm{b}}\right]$} & {$\left[0.0147^{\mathrm{a}}, 0.019^{\mathrm{b}}\right]$} & {$\left[3.8^{\mathrm{a}}, 5.7^{\mathrm{b}}\right],(7.5 \pm 6.5)^{\mathrm{c}}$} \\
\hline Sea salt & 4.85 & 0.022 & 2.9 \\
\hline & {$\left[3.94^{\mathrm{a}}, 6.43^{\mathrm{b}}\right]$} & {$\left[0.032^{\mathrm{a}}, 0.030^{\mathrm{b}}\right]$} & {$\left[4.1^{\mathrm{a}}, 3.0^{\mathrm{b}}\right]$} \\
\hline Dust & 13.08 & 0.013 & 0.71 \\
\hline & {$\left[22.9^{\mathrm{a}}, 19.9^{\mathrm{b}}\right]$} & {$\left[0.021^{\mathrm{a}}, 0.032^{\mathrm{b}}\right]$} & {$\left[0.47^{\mathrm{a}}, 0.95^{\mathrm{b}}\right]$} \\
\hline
\end{tabular}

a 2010 values from GEOS-Chem chemical transport model, with GFED3 fire emissions (Heald et al., 2014)

${ }^{\mathrm{b}}$ AEROCOM I medians from Kinne et al. (2006)

${ }^{c}$ AEROCOM II means from Myhre et al. (2013) 
(a) Porto Velho

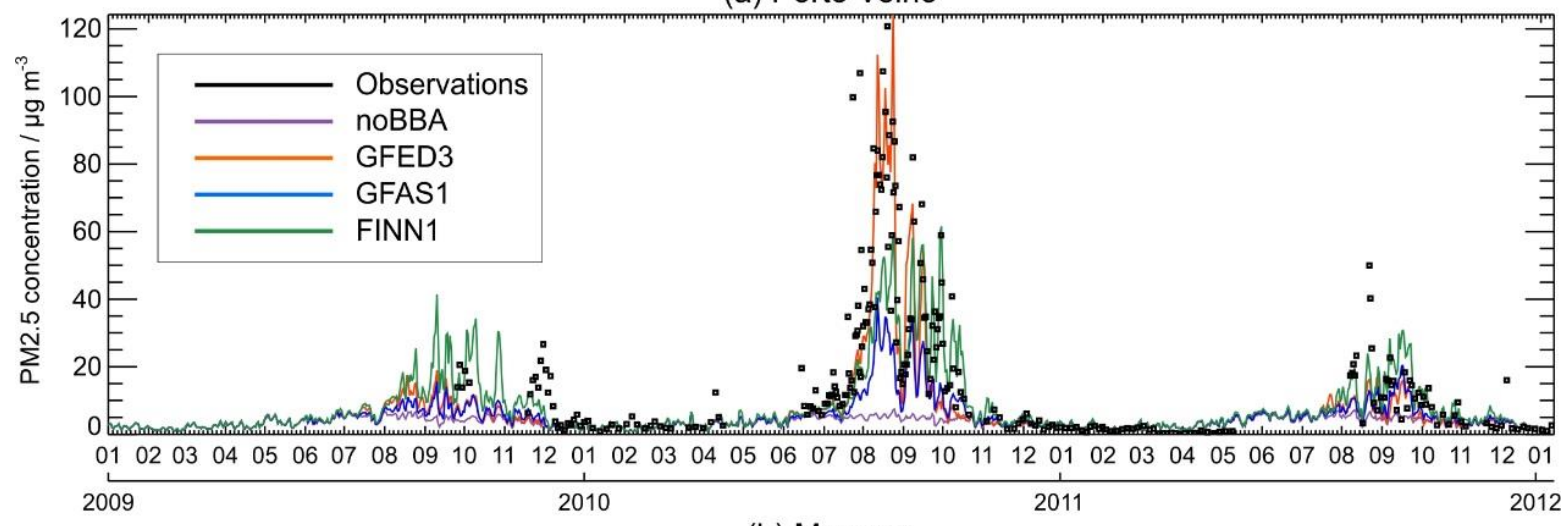

(b) Manaus

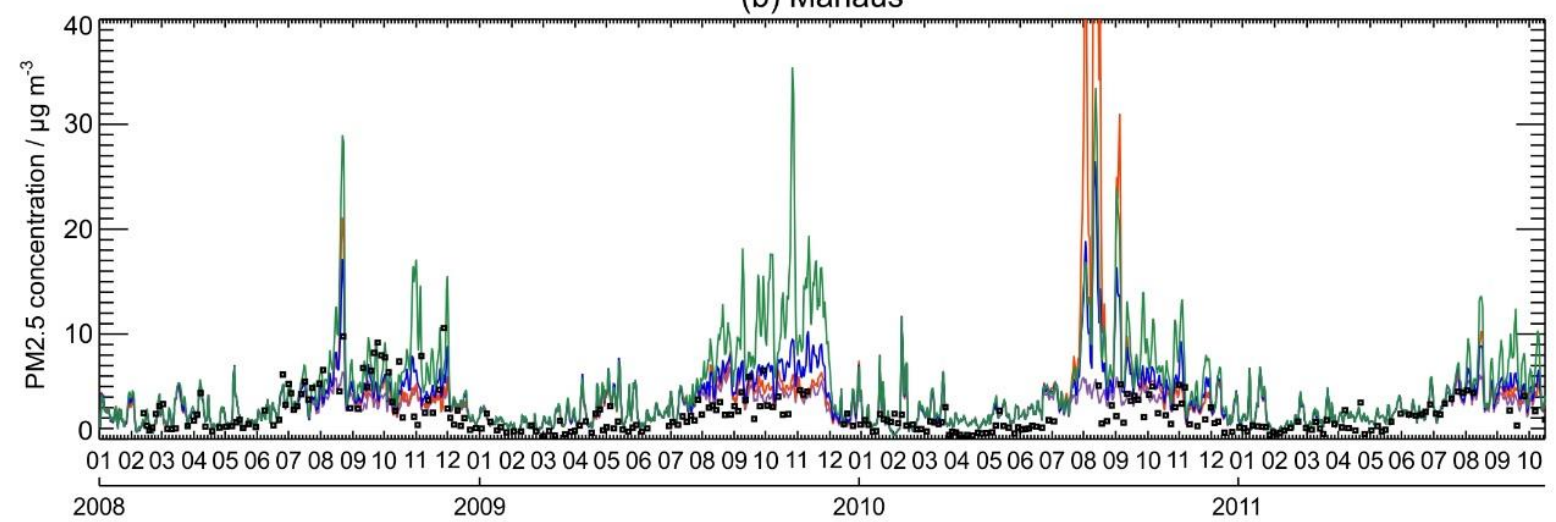

(c) Santarem

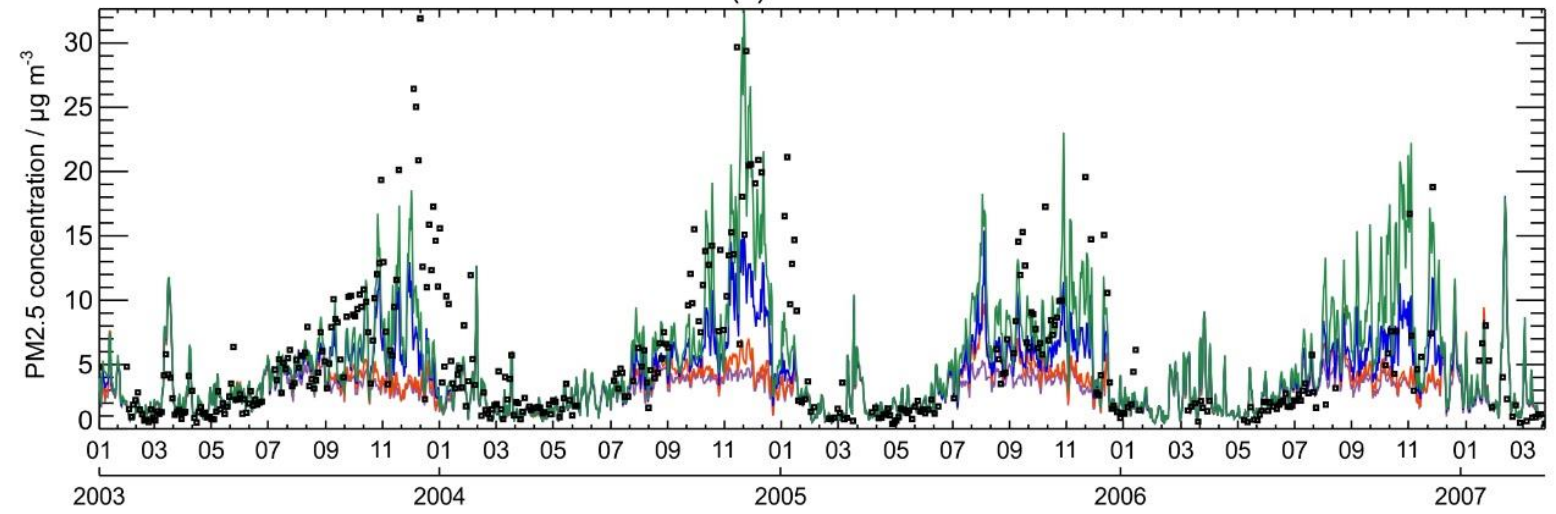

(d) Alta Floresta

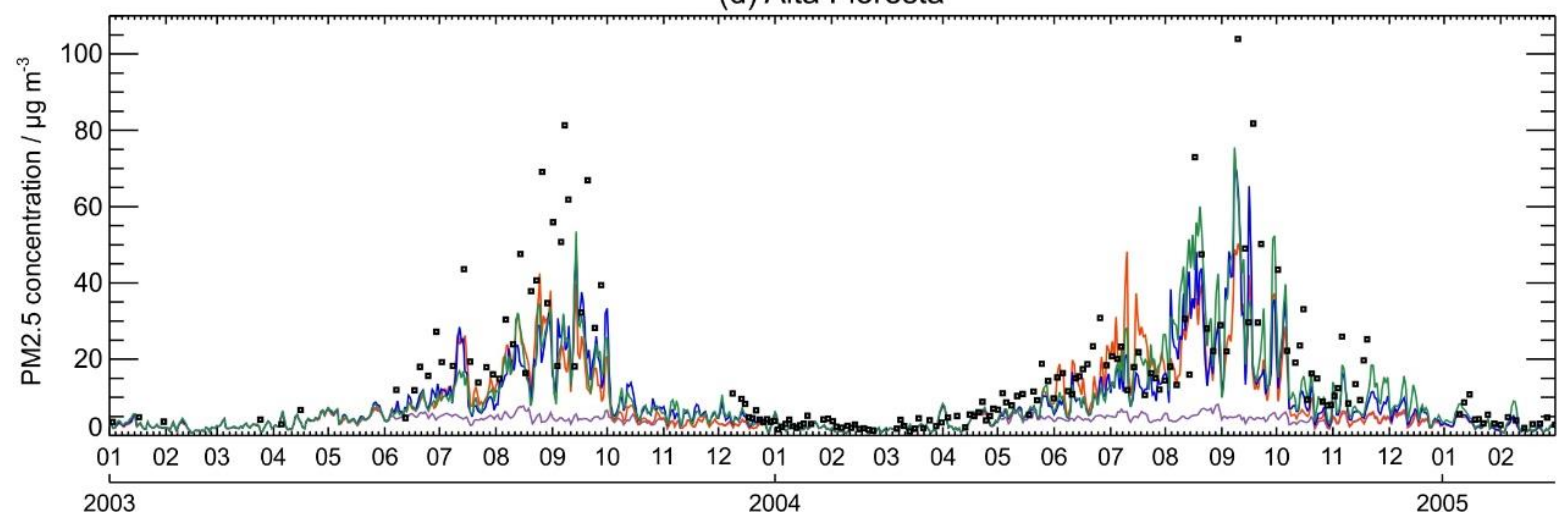

Figure S1. Time-series of observed (black) and simulated (colour) PM2.5 concentrations at four ground stations in the Amazon region: (a) Porto Velho (2009-2011); (b) Manaus (2008-2011); (c) Santarem (2003-2006); and (d) Alta Floresta (2003-2004). The model PM2.5 concentrations are daily averages. 
The time resolution of the observed PM2.5 concentrations depends on the measurement duration, which ranged from less than 1 day to more than 10 days. Thus the observation data points represent averages over a range of time periods. The modelled results are shown for four simulations: without biomass burning (purple), with GFED3 emissions (red), with GFAS1 emissions (blue) and with FINN1 emissions (green).

(a) noBBA

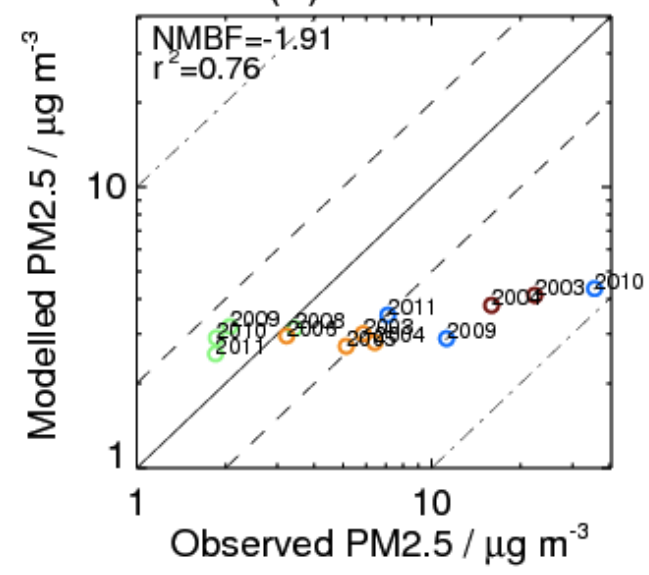

(c) GFAS1

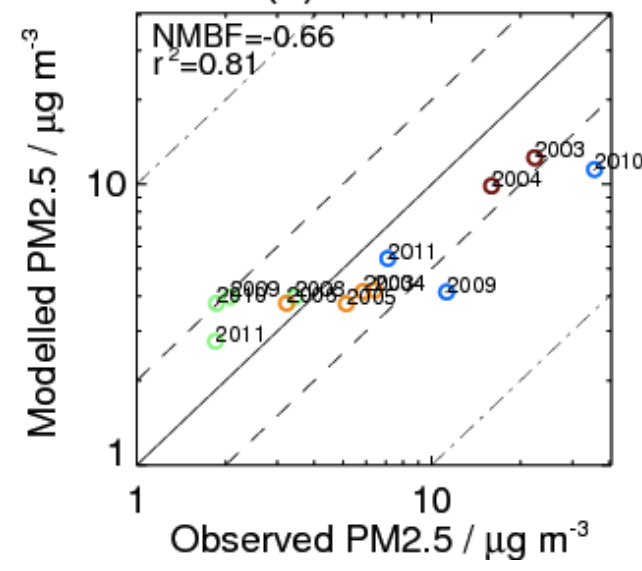

(b) GFED3

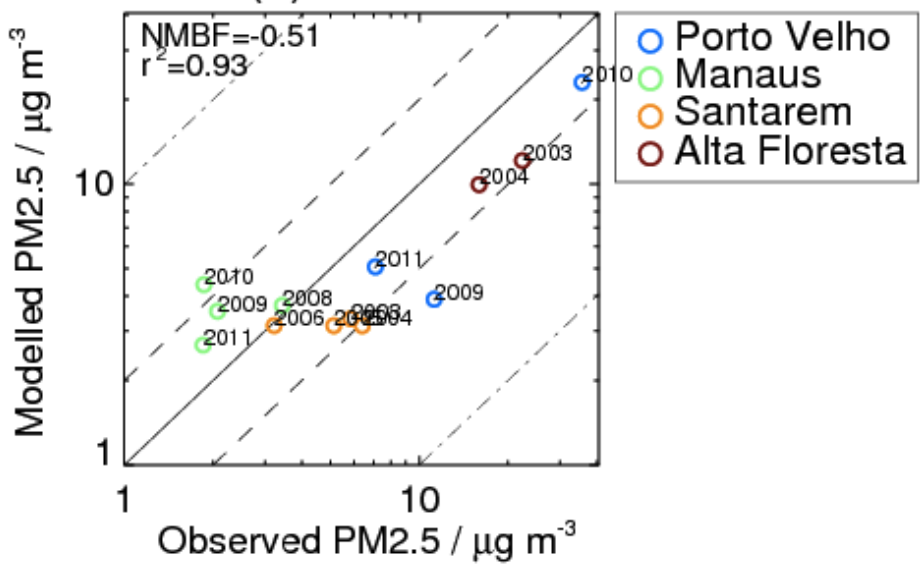

(d) FINN1

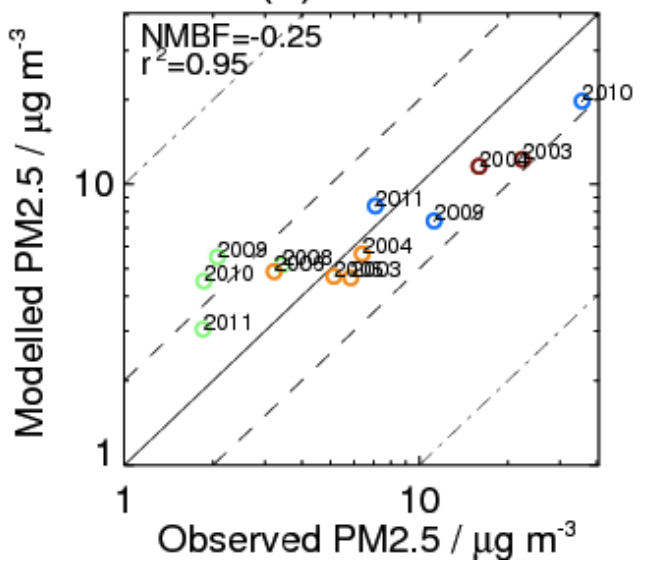

Figure S2. Simulated versus observed annual mean PM2.5 concentrations at each ground station in the Amazon region for the model (a) without biomass burning emissions; and with (b) GFED3; (c) GFAS1; and (d) FINN1 emissions. The modelled and observed annual mean concentrations are calculated for every year of available data between 2003 and 2011 (inclusive). The normalised mean bias factor (NMBF; Yu et al., 2006) and Pearson's correlation $\left(\mathrm{r}^{2}\right)$ between modelled and observed PM2.5 concentrations are shown in the top left corner. 
(a) noBBA

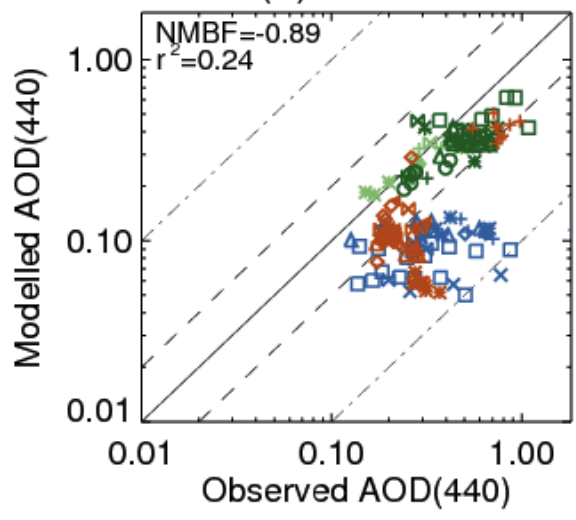

(c) GFAS1

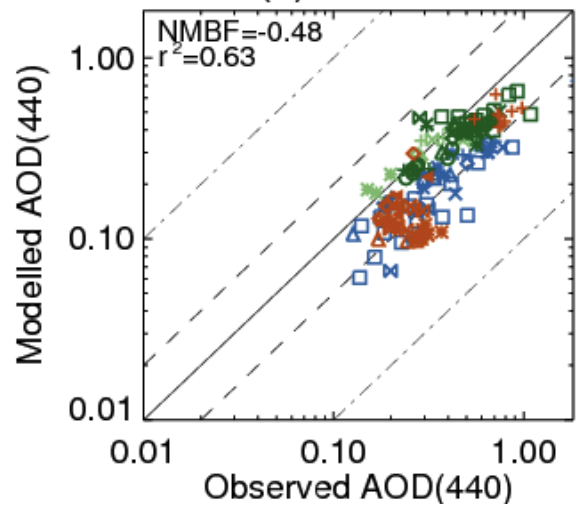

(b) GFED3

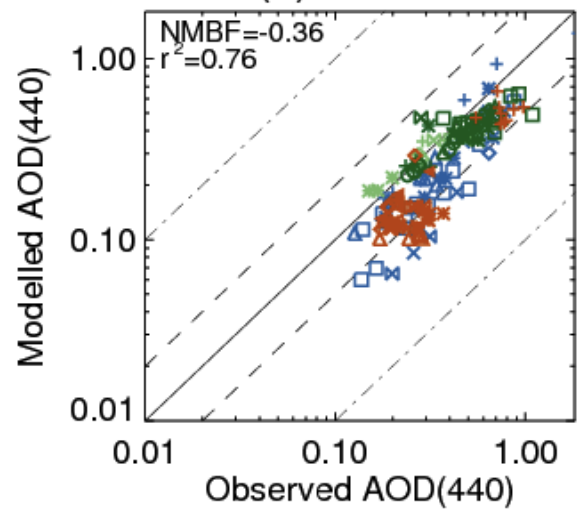

(d) FINN1

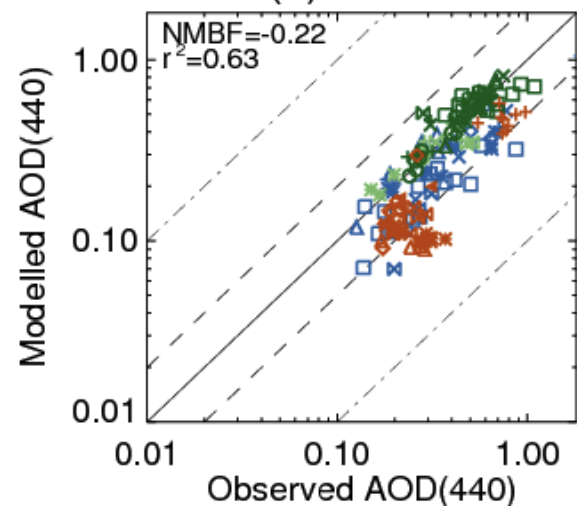

+ Ji_Parana_SE

* Alta Floresta

$\bowtie$ Belterra

$\diamond$ Abracos_Hill

$\triangle$ Rio Branco

- CUIABA-MIRANDA

$\times$ SANTA CRUZ UTEPSA

$\square$ SANTA CRUZ

+ Singapore

* ND_Marbel_Univ

$\bowtie$ Bañdung

$\checkmark$ Manila Observatory

+ Songkh̄la_Met_Sta

* Chulalongkorn

$\bowtie$ Ubon_Ratchathani

$\diamond$ Silpakorn_Univ

$\triangle$ Chiang_Mai_Met_Sta

Mukdahan

$\times$ Pimai

$\square$ Bac Giang

○ Bac_Lieu

+ Ilorin

* Mongu

$\bowtie$ Skukuza

$\diamond$ Wits_University

$\triangle$ ICIPE-Mbita

4 Ascension_Island

Figure S3. Simulated versus observed annual mean AOD at $440 \mathrm{~nm}$ at each AERONET station. The model is shown (a) without biomass burning emissions; and with (b) GFED3; (c) GFAS1; and (d) FINN1 emissions. The modelled and observed annual mean AODs are calculated from daily mean data, for every year of available data between 2003 and 2011 (inclusive). AERONET stations located in South America are shown in blue; stations in Southeast Asia are shown in green (stations in Equatorial Asia and Indochina in light and dark green, respectively); and stations in Africa are shown in orange. The normalised mean bias factor (NMBF) and Pearson's correlation $\left(\mathrm{r}^{2}\right)$ between modelled and observed PM2.5 concentrations are shown in the top left corner. 
(a) noBBA

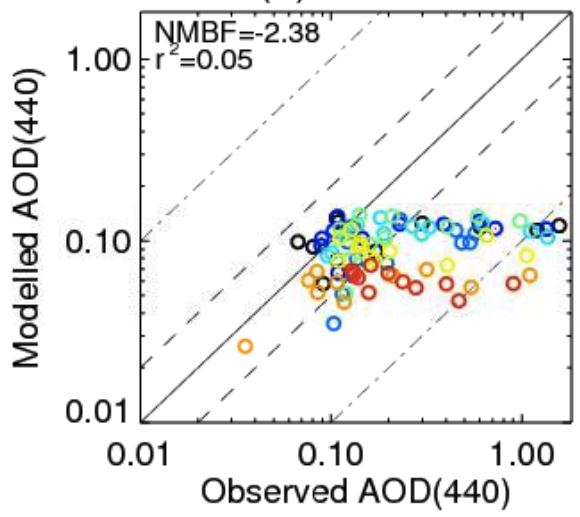

(c) GFAS1

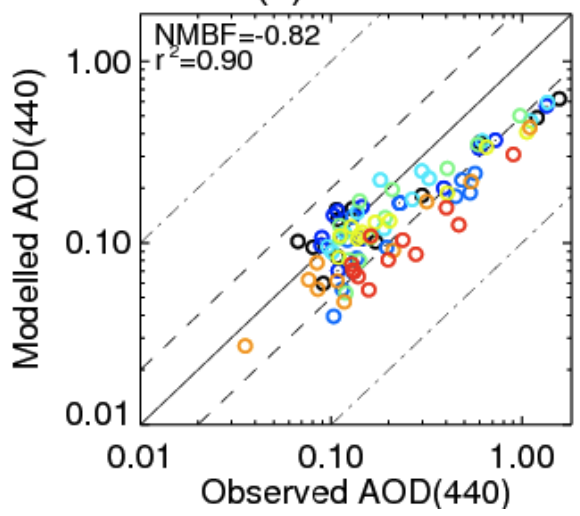

(b) GFED3

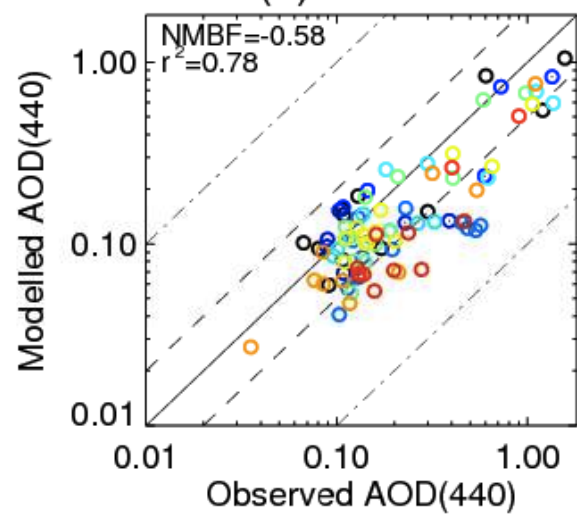

(d) FINN1

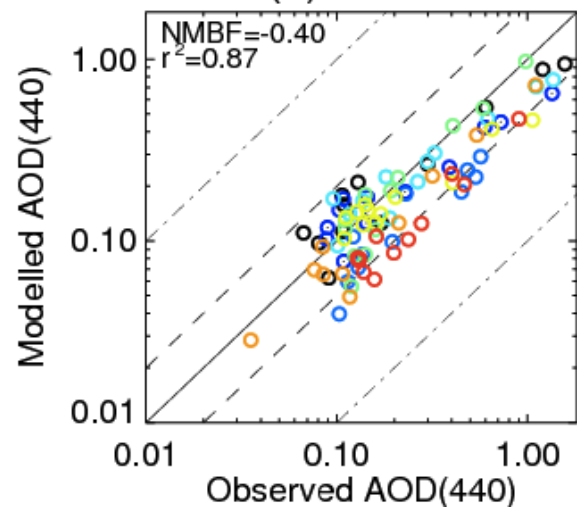

Figure S4. Simulated versus observed multi-annual monthly mean AOD at $440 \mathrm{~nm}$ at each of the AERONET stations located in South America. The model is shown (a) without biomass burning emissions; and with (b) GFED3; (c) GFAS1; and (d) FINN1 emissions. The multi-annual monthly mean AODs were calculated using all years of daily mean data available between January 2003 and December 2011 to obtain an average seasonal cycle at each station. The normalised mean bias factor (NMBF) and Pearson's correlation $\left(\mathrm{r}^{2}\right)$ between modelled and observed PM2.5 concentrations are shown in the top left corner. 
(a) Internal + ZSR

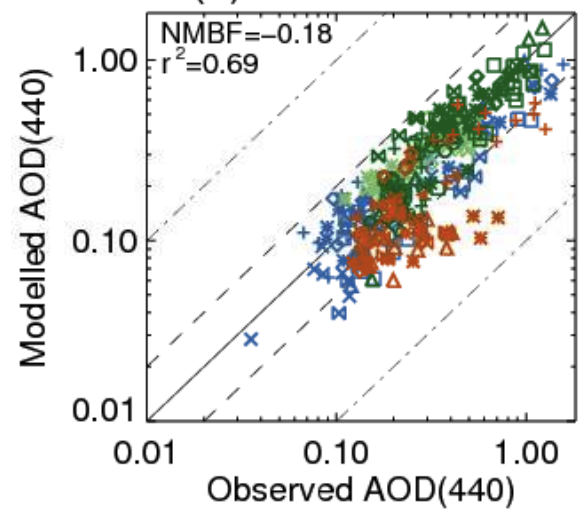

(c) Internal $+\kappa-$ Köhler

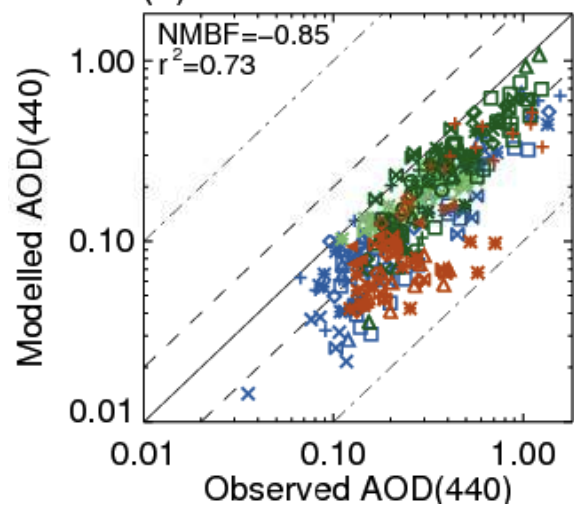

(b) External + ZSR

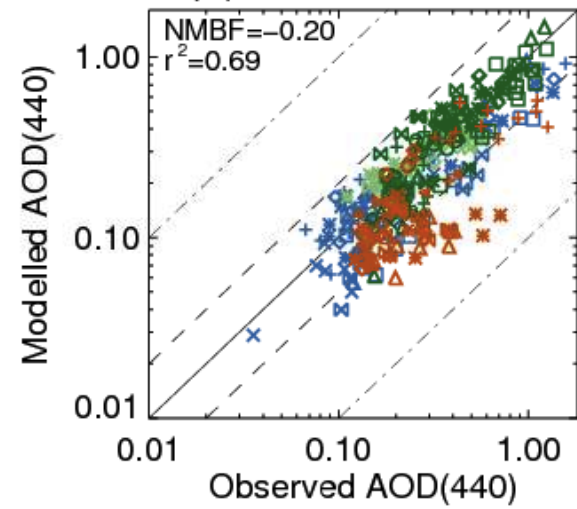

(d) External $+\kappa-$ Köhler

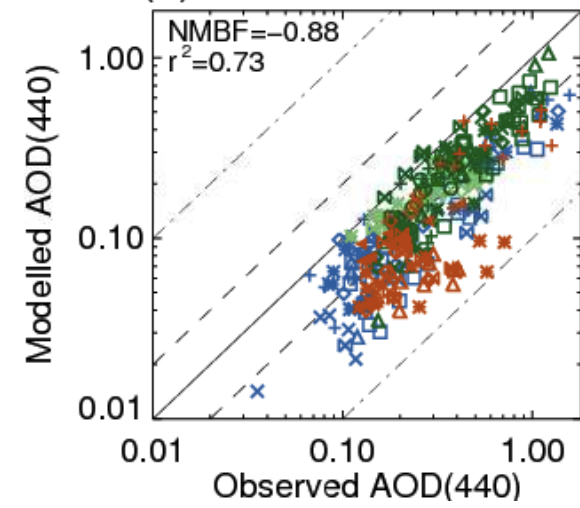

+ Ji_Parana_SE

* Alta_Floresta

$\bowtie$ Belterra

$\diamond$ Abracos_Hill

$\triangle$ Rio Branco

$\square$ CUIABA-MIRANDA

$\times$ SANTA_CRUZ UTEPSA

$\square$ SANTA CRUZ

+ Singapore

* ND_Marbel_Univ

$\bowtie$ Bandung

$\checkmark$ Manila_Observatory

+ Songkh̄la_Met_Sta

* Chulalongkorn

$\bowtie$ Ubon_Ratchathani

$\diamond$ Silpakorn Univ

$\triangle$ Chiang_Mai_Met_Sta

$\square$ Mukdahan

$\times$ Pimai

$\square$ Bac_Giang

o Bac_Lieu

+ llorin

* Mongu

$\bowtie$ Skukuza

$\diamond$ Wits_University

$\triangle$ ICIP $\bar{E}-$ Mbita

$\triangleleft$ Ascension_Island

Figure S5. Simulated versus observed multi-annual monthly mean AOD at $440 \mathrm{~nm}$ at each AERONET station to demonstrate the sensitivity of simulated AOD to different assumptions. The model is with FINN1 fire emissions and simulated AOD is calculated assuming (a) internal mixing with ZSR water uptake scheme (identical to Fig. 5d); (b) external mixing with ZSR water uptake scheme; (c) internal mixing with $\kappa$-Köhler water uptake scheme; and (d) external mixing with $\kappa$-Köhler water uptake scheme. AERONET stations located in South America are shown in blue; stations in Southeast Asia are shown in green (stations in Equatorial Asia and Indochina in light and dark green, respectively); and stations in Africa are shown in orange. The normalised mean bias factor (NMBF) and Pearson's correlation (r2) between modelled and observed PM2.5 concentrations are shown in the top left corner. 


\section{References}

Heald, C. L., Ridley, D. A., Kroll, J. H., Barrett, S. R. H., Cady-Pereira, K. E., Alvarado, M. J., and Holmes, C. D.: Contrasting the direct radiative effect and direct radiative forcing of aerosols, Atmos. Chem. Phys., 14, 5513-5527, doi:10.5194/acp-14-5513-2014, 2014.

Kinne, S., Schulz, M., Textor, C., Guibert, S., Balkanski, Y., Bauer, S. E., Berntsen, T., Berglen, T. F., Boucher, O., Chin, M., Collins, W., Dentener, F., Diehl, T., Easter, R., Feichter, J., Fillmore, D., Ghan, S., Ginoux, P., Gong, S., Grini, A., Hendricks, J., Herzog, M., Horowitz, L., Isaksen, I., Iversen, T., Kirkevåg, A., Kloster, S., Koch, D., Kristjansson, J. E., Krol, M., Lauer, A., Lamarque, J. F., Lesins, G., Liu, X., Lohmann, U., Montanaro, V., Myhre, G., Penner, J., Pitari, G., Reddy, S., Seland, O., Stier, P., Takemura, T., and Tie, X.: An AeroCom initial assessment - optical properties in aerosol component modules of global models, Atmos. Chem. Phys., 6, 1815-1834, doi:10.5194/acp-6-1815-2006, 2006.

Myhre, G., Samset, B. H., Schulz, M., Balkanski, Y., Bauer, S., Berntsen, T. K., Bian, H., Bellouin, N., Chin, M., Diehl, T., Easter, R. C., Feichter, J., Ghan, S. J., Hauglustaine, D., Iversen, T., Kinne, S., Kirkevag, A., Lamarque, J. F., Lin, G., Liu, X., Lund, M. T., Luo, G., Ma, X., van Noije, T., Penner, J. E., Rasch, P. J., Ruiz, A., Seland, O., Skeie, R. B., Stier, P., Takemura, T., Tsigaridis, K., Wang, P., Wang, Z., Xu, L., Yu, H., Yu, F., Yoon, J. H., Zhang, K., Zhang, H., and Zhou, C.: Radiative forcing of the direct aerosol effect from AeroCom Phase II simulations, Atmos. Chem. Phys., 13, 1853-1877, doi:10.5194/acp-13-1853-2013, 2013.

Petters, M. D. and Kreidenweis, S. M.: A single parameter representation of hygroscopic growth and cloud condensation nucleus activity, Atmos. Chem. Phys., 7, 1961-1971, doi:10.5194/acp7-1961-2007, 2007.

Reid, J. S. and Hobbs, P. V.: Physical and optical properties of smoke from individual biomass fires in Brazil, J. Geophys. Res., 103, 32 013-32 031, 1998. 